\title{
Semiempirical local-field correction function for electrons in Li metal
}

\author{
W. Schülke, K. Höppner, and A. Kaprolat \\ Institute of Physics, University of Dortmund, D-44221 Dortmund, Germany
}

(Received 29 April 1996; revised manuscript received 29 July 1996)

\begin{abstract}
A frequency average of the complex local-field correction function (LFCF), $G(q, \bar{\omega})$, of electrons in $\mathrm{Li}$ metal has been semiempirically determined for $1.12 k_{F} \leqslant q \leqslant 2.59 k_{F}\left(k_{F}=\right.$ Fermi momentum) by fitting experimental dynamic structure factors, $S(\mathbf{q}, \omega)$, to jellium $S(q, \omega)$. The range of frequency averaging was between 3 and $4 \mathrm{eV}$ (fitting range), its position was in the low-frequency region between 3 and $9 \mathrm{eV}$, depending on $q$. For $q<1.6 k_{F}$, the real part of the semiempirical $\operatorname{LFCF}, \operatorname{Re} G(q, \bar{\omega})$, is consistent with all current theories, when compared with the static LFCF $G(q, \omega=0)$. For $q>1.6 k_{F}$, the semiempirical $\operatorname{Re} G(q, \bar{\omega})$ exhibits a strong increase in values ranging from 1.2 to 2.4 in a manner which has not been predicted by any existing theory. The relation between $G(q, 0)$ and the momentum distribution function $n(k)$ is stressed to make contact with a most recently found anomalous behavior of $n(k)$ in Li. [S0163-1829(96)09647-6]
\end{abstract}

\section{INTRODUCTION}

The dynamic local-field correction function (LFCF), $G(\mathbf{q}, \omega),{ }^{1}$ whose static approximation has been first introduced by Hubbard ${ }^{2}$ is an important quantity for the understanding of exchange- and correlation-induced dynamic and static properties of electron liquids. A great deal of theoretical work has been invested to find expressions of the static LFCF, $G(q, 0)$, for homogeneous systems, which satisfy simultaneously the compressibility sum rule, all frequency moment sum rules, and the non-negative condition of the pair-correlation function $g(r)$ at the origin. We will not refer to all these calculations and point only to significant differences of their results with respect to both their behavior near $q=2 k_{F} \quad\left(k_{F}=\right.$ Fermi momentum $)$, and their limits of short wavelengths. All authors agree with a quadratic dependence of $G(q, 0)$ for small $q$, which is necessary to satisfy the compressibility sum rule and which is found in the very early expressions of Hubbard ${ }^{2}$ and of Geldart and Vosko. ${ }^{3}$ Brosens et $a l^{4}$ and Holas et al. ${ }^{5}$ discovered a more or less pronounced peak of $G(q, 0)$ around $2 k_{F}$ with values clearly above one, whereas Geldart and Taylor ${ }^{6}$ failed to see this peak in their calculations. Also Utsumi and Ichimaru ${ }^{7}$ found this peak but substantially moderated compared to Ref. 4. Concerning the limit of short wavelength it is very often believed that the relation

$$
\lim _{q \rightarrow \infty} G(q, 0)=(2 / 3)[1-g(0)],
$$

first introduced by Niklasson, ${ }^{8}$ holds, so that $G(q, 0)$ must reach a constant value smaller than 1 for large $q$, since $g(r)$ has to be positive definite. Also the analytical expression for $G(q, 0)$ of Utsumi and Ichimaru, ${ }^{7}$ very often used in the literature, exhibits this limiting property. But it has been pointed out by Holas $^{9}$ that Eq. (1) is only valid if the Lindhard dielectric function, ${ }^{10}$ used in the defining equation for $G(q, \omega)$, is a modified one with the momentum distribution function of noninteracting electrons, $n_{0}(k)=\theta\left(1-k / k_{F}\right)$, replaced by that of interacting ones, $n_{I}(k)$. It has been shown by Holas, ${ }^{9}$ that using the nonmodi- fied Lindhard dielectric function leads to a large- $q$ expansion of $G(q, 0)$, which contains, besides the right-hand side of Eq. (1), also contributions quadratic in $q$, so that values larger than 1 can be reached for sufficiently large $q$ 's. Farid et al. ${ }^{11}$ have derived an expression for $G(q, 0)$, which contains, as leading parameters, the relative changes of, respectively, the mean kinetic energy and the mean-squared kinetic energy that occur when going from a noninteracting to an interacting electron system, so that $G(q, 0)$ is directly related to $n_{I}(k)$, the momentum distribution function of an interacting electron system. We will make use of this relation in what follows. Even after the clarification, carried out by Holas, ${ }^{9}$ the situation on the theoretical front remains rather confusing, as indicated by the different results of very recent calculations, although these treatments claim to satisfy the first and third frequency moment sum rule, the compressibility sum rule, and the nonnegative condition of $g(0)$. As an example, compare $G(q, 0)$ of Ref. 11 with that of Ref. 12 . It seems to be difficult to find additional criteria for the validity of approximations performed in those calculations.

For this reason we decided to introduce experimental input into the discussion by utilizing experimental results for $S(\mathbf{q}, \omega)$, the dynamic structure factor ${ }^{13,14}$ of the valence electrons, since $S(\mathbf{q}, \omega)$ is heavily influenced by the LFCF, so that one can extract information on the LFCF from these experimental data in a semiempirical way. The term "semiempirical" means that the LFCF can be obtained from experimental data only, if theoretical data for $S(\mathbf{q}, \omega)$ are at hand, calculated within the limits of the random phase approximation (RPA). In a first approach, we intend to look at the $q$ dependence of the LFCF, where we will perform a frequency average within a $3-4 \mathrm{eV}$ wide frequency range in the low-frequency region.

The rest of this paper is organized as follows. In Sec. II we present the basic idea of how the semiempirical determination of a LFCF can be performed. The measurements of $S(\mathbf{q}, \omega)$ and details of the fitting procedure, which leads to the semiempirical values of the LFCF, are described in Sec. III. In Sec. IV the results are discussed and final conclusions are drawn. 


\section{BASIC RELATIONS FOR THE SEMIEMPIRICAL DETERMINATION OF THE LFCF}

We will use the definition of $G(\mathbf{q}, \omega)$, as first proposed by Hubbard $^{2}$ by writing the dielectric function $\varepsilon(\mathbf{q}, \omega)$ of an interacting electron system as follows:

$$
\begin{aligned}
\varepsilon(\mathbf{q}, \omega)= & +\left[\varepsilon^{\mathrm{RPA}}(\mathbf{q}, \omega)-1\right]\{1-G(\mathbf{q}, \omega) \\
& \left.\times\left[\varepsilon^{\mathrm{RPA}}(\mathbf{q}, \omega)-1\right]\right\}^{-1},
\end{aligned}
$$

where $\varepsilon^{\mathrm{RPA}}(\mathbf{q}, \omega)$ is the dielectric function calculated within the limits of the random phase approximation (RPA). For a homogeneous electron system this is the well-known Lindhard dielectric function. ${ }^{10}$ For inhomogeneous systems we have to insert the Ehrenreich-Cohen dielectric function ${ }^{15}$ into Eq. (2). By doing so, one neglects nondiagonal elements of the dielectric matrix. ${ }^{16}$ As already pointed out in Sec. I, Farid et al. ${ }^{11}$ have stressed the relationship between $n_{I}(k)$, the momentum distribution function of an interacting electron system, and $G(q, 0)$. Their equations for $G(q, 0)$ [Eqs. (30a)-(30e) and (31a)-(31e) of Ref. 11], which should not be reproduced here, contain, as leading parameters, the relative changes $\delta_{2}$ and $\delta_{4}$ of, respectively, the mean kinetic energy and the mean squared kinetic energy, which occur when going from a noninteracting to an interacting electron system. Thus $\delta_{2}$ and $\delta_{4}$ can be expressed in terms of $n_{0}(k)$ and $n_{I}(k)$, respectively, in the following way:

$$
\delta_{l} \equiv\left(\left\langle k^{l}\right\rangle_{I}-\left\langle k^{l}\right\rangle_{0}\right) /\left\langle k^{l}\right\rangle_{0},
$$

where

$$
\left\langle k^{l}\right\rangle_{I, 0} \equiv\left(3 / k_{F}^{3}\right) \int_{0}^{\infty} d k k^{2} n_{I, 0}(k) k^{l},
$$

so that $G(q, 0)$ is directly related to $n_{I}(k)$. We will make use of this relation in what follows.

The semiempirical determination of LFCF, as proposed in this paper, is based on fitting measured dynamic structure factors, $S(\mathbf{q}, \omega)$, to calculated ones. According to both the following relation between $S(\mathbf{q}, \omega)$ and the dielectric function $\varepsilon(\mathbf{q}, \omega)$,

$$
\begin{gathered}
S(\mathbf{q}, \omega) \equiv-\left(\hbar q^{2} / 4 \pi^{2} e^{2} n\right) \operatorname{Im} \varepsilon^{-1}(\mathbf{q}, \omega), \\
n=\text { electron density, }
\end{gathered}
$$

and Eq. (2), $S(\mathbf{q}, \omega)$, must be heavily influenced by the correct choice of $G(\mathbf{q}, \omega)$. Since $G(\mathbf{q}, \omega)$ is in general complex for nonzero frequencies, one cannot obtain $G(\mathbf{q}, \omega)$ directly from Eqs. (2) and (5). The way one would proceed in order to extract $G(\mathbf{q}, \omega)$ for every frequency separately would consist in making a Kramers-Kronig analysis of the measured $S(\mathbf{q}, \omega)$, thus getting expressions for $\operatorname{Re} \varepsilon(\mathbf{q}, \omega)$ and $\operatorname{Im} \varepsilon(\mathbf{q}, \omega),{ }^{17}$ which can be used to end up with both $\operatorname{Re} G(\mathbf{q}, \omega)$ and $\operatorname{Im} G(\mathbf{q}, \omega)$. But difficulties with the correct treatment of the high-frequency range because of the superposition of the valence electron part with the $1 s$-core part of the inelastic spectrum for $\hbar \omega>55 \mathrm{eV}$ in the case of $\mathrm{Li}$, make this type of analysis less reliable. For these reasons we de- cided to fit, within a certain frequency range, calculated $S(\mathbf{q}, \omega)$ to experimental ones. The calculations of $S(q, \omega)$ have to be performed by means of a complex frequencyaveraged LFCF, $G(\mathbf{q}, \bar{\omega})$, where frequency averaging refers to the fitting range. The fitting range should be small enough, in order to account for the frequency dependence of $G(\mathbf{q}, \omega)$, and should be close to $\omega=0$, in order to make possible comparison with theoretical calculations of the static limit of the LFCF, $G(q, \omega=0)$. This definition of a frequency-averaged LFCF enables a rather straightforward determination of this quantity from experimental data: One has to least-square fit calculated $S(\mathbf{q}, \omega)$ to experimental ones within the frequency range $\bar{\omega} \pm \Delta \omega$, that defines the frequency average of $G(\mathbf{q}, \omega)$ by independently varying $\operatorname{Re} G(\mathbf{q}, \bar{\omega})$ and $\operatorname{Im} G(\mathbf{q}, \bar{\omega})$, respectively. The choice of an appropriate frequency range $\bar{\omega} \pm \Delta \omega$ should satisfy two demands:

(i) As already pointed out, $\omega$ should be in the lowfrequency region, in order to accomplish comparison with calculated $G(q, \omega=0)$, since most of the theoretical calculations have been done in this limit.

(ii) Within the frequency range $\bar{\omega} \pm \Delta \omega$, lattice-induced fine structure of $S(\mathbf{q}, \omega)$ should be at minimum over the whole range of $q$ under investigation. It has been shown by Schülke et al. ${ }^{18}$ on the basis of pseudopotential bandstructure calculations that the overall shape of the structure factor $S(\mathbf{q}, \omega)$ of Li single crystals, plotted as a function of $\omega$, is independent of the $\mathbf{q}$ direction and agrees with the jellium-model-calculated $S(q, \omega)$. This overall shape is modulated by a q-orientation-dependent fine structure, which can be attributed to energy gaps of unoccupied states at zone boundaries in the extended zone scheme, and which turned out to be the only lattice effect. But also $a b$ initio local-density approximation (LDA) calculations of $S(\mathbf{q}, \omega)$ for a simple metal such as ${ }^{19} \mathrm{Al}$ have clearly shown (see, for instance, Fig. 3 of Ref. 19) that the lattice-induced fine structure of $S(\mathbf{q}, \omega)$ consists in a modulation of the jellium $S(q, \omega)$, which leaves the overall shape untouched. Therefore, it seems to be justified to found the fit on jelliumcalculated dynamic structure factors, since, as shown below, one can find a frequency region, where $S(\mathbf{q}, \omega)$ is both hardly influenced by the lattice-induced fine structure and not superimposed by the core contribution. According to Ref. 18, the lattice-induced modulation was weakest in the [111] direction of $\mathbf{q}$. Hence we have chosen this direction for taking our experimental data. Moreover, the only latticeinduced fine structure in the [111] direction consists in a dip, which is roughly $10 \mathrm{eV}$ wide and is positioned around $16 \mathrm{eV}$ with only weak dispersion. Taking additionally into account that the core contribution to $S(\mathbf{q}, \omega)$ starts at $55 \mathrm{eV}$, the $\mathrm{Li}-\mathrm{K}$-binding energy, the frequency range between 2 and 12 $\mathrm{eV}$ is best suited for fitting jellium-calculated $S(q, \omega)$ to the experiment. (Experimental results for frequencies smaller than $2 \mathrm{eV}$ are less reliable because of the strong superposition with the quasielastically scattered line in the rough data.) Use of the pure RPA jellium model $S(q, \omega)$ might be even more advisable than being based on band-structurecalculated dynamical response functions, whenever the bandstructure scheme used includes exchange and correlation in any type of approximation. The defining Eq. (2) asks for band-structure data in the Ehrenreich-Cohen dielectric 

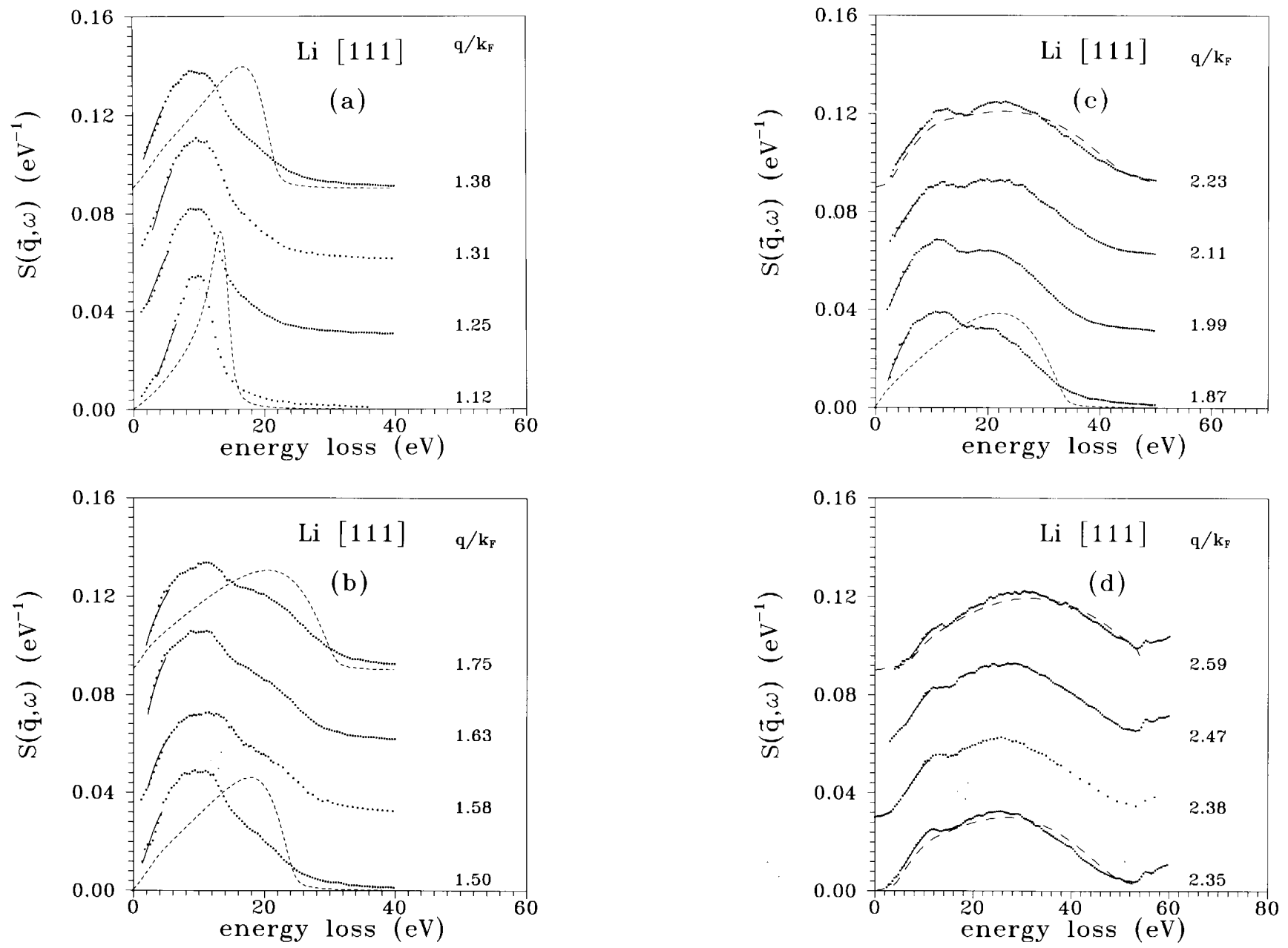

FIG. 1. (a)-(d) Data points: experimental dynamic structure factors $S(\mathbf{q}, \omega)$ of Li for $1.12 k_{F} \leqslant q \leqslant 2.59 k_{F}$, q $\|[111]$; solid line, jellium $S(q, \omega)$ within the fitting range of frequency, calculated with the optimum $G(q, \bar{\omega})$; short-dashed line, RPA jellium $S(q, \omega)$; long-dashed line, jellium $S(q, \omega)$, calculated with $G(q, 0)$ of Ref. 7 .

function ${ }^{15}$ that have to be calculated using an exchange- and correlation-free crystal potential (Hartree approximation).

It must be stressed that in general the frequency-averaged LFCF, $G(\mathbf{q}, \bar{\omega})$, might be different from the static LFCF, $G(q, \omega=0)$. Therefore, the comparison of fitted $\operatorname{Re} G(q, \bar{\omega})$ values with theoretical $G(q, 0)$ is subject to an uncertainty, which can be estimated to be of the same order of magnitude as the variation of calculated $\operatorname{Re} G(q, \bar{\omega})$ within the fitting frequency range, shown e.g., in Ref. 20. For $q=k_{F}$ these calculations have shown that $G(q, 0)$ is nearly equal to the average value of $\operatorname{Re} G(q, \bar{\omega})$, taken over the frequency range of our fitting procedure.

To sum up, we have fitted, within a certain frequency range $\bar{\omega} \pm \Delta \omega$, experimental $S(\mathbf{q}, \omega)$ for $\mathbf{q} \|[111]$ to localfield-corrected Lindhard $S(q, \omega)$ by varying independently the real and imaginary part of a frequency-averaged LFCF, $G(q, \bar{\omega})$, in order to get $G(q, \bar{\omega})$ that optimally fits the experiment within this frequency range.

\section{MEASUREMENT AND FITTING PROCEDURE}

We have measured the double-differential scattering cross section DDSCS of single-crystal Li metal with $\mathbf{q} \|[111]$ for 18 different values of $q>q_{c} \quad\left(q_{c}=0.46\right.$ a.u. for $\mathrm{Li}$; a.u. =atomic units: $\hbar=e=m=1$ ) between $1.12 k_{F}$ and $2.59 k_{F}$ $\left(k_{F}=\right.$ Fermi momentum $=0.58$ a.u. for a homogeneous electron system with the valence electron density of Li) with 1 $\mathrm{eV}$ resolution using synchrotron radiation from the DORISstorage ring at DESY, Hamburg, monochromatized to 7.99 $\mathrm{keV}$ by means of a $\mathrm{Ge}(311)$ double-crystal monochromator, and by employing a $\mathrm{Si}(444)$ spherically bent analyzer. The experimental setup used is described in detail elsewhere. ${ }^{21}$ The data processing for getting $S(\mathbf{q}, \omega)$ from the measured DDSCS is presented in Ref. 18. Selected experimental $S(\mathbf{q}, \omega)$ are plotted in Figs. 1(a)-1(d). One can easily verify that there exists in all spectra a dip, which is roughly $10 \mathrm{eV}$ wide and is positioned between 15 and $18 \mathrm{eV}$, depending on $q$. This dip has proved to be a lattice-induced effect, since its strength is q-orientation dependent. ${ }^{18}$ When going from $q=1.25 k_{F}$ to $2.59 k_{F}$, the position of this lattice-induced dip changes from the high- $\omega$ half of $S(\mathbf{q}, \omega)$ to its center, leaving the lower- $\omega$ flank untouched. Therefore, the fitting of the experimental $S(\mathbf{q}, \omega)$ to the calculated ones was performed at the low- $\omega$ flank of the $S(\mathbf{q}, \omega)$ curves. For this purpose we have defined the frequency range $\bar{\omega} \pm \Delta \omega$ for the least-square fitting of experimental $S(\mathbf{q}, \omega)$ to theoretical ones in the following way: If $I_{\max }$ was the absolute maximum value of the $S(\mathbf{q}, \omega)$ spectrum, 


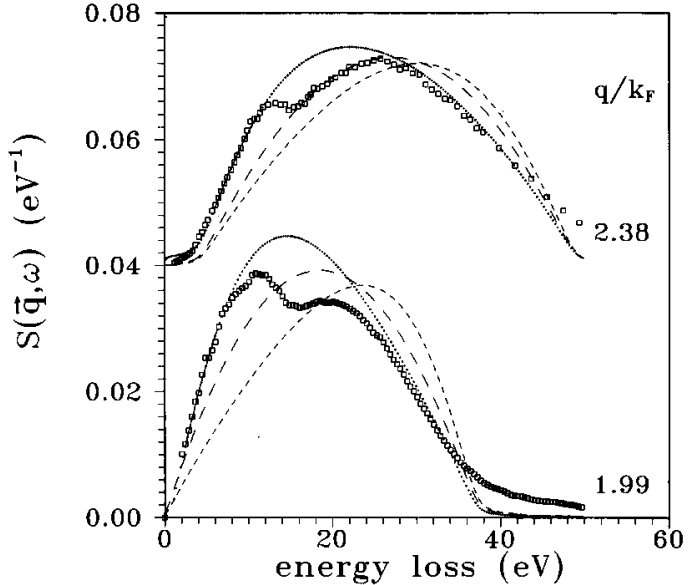

FIG. 2. Data points experimental $S(\mathbf{q}, \omega)$ of Li for $q=1.99 k_{F}$ and $q=2.38 k_{F}$, respectively; $\mathbf{q} \|[111]$; solid line, jellium $S(q, \omega)$ within the fitting range of frequency, calculated with optimum $G(q, \bar{\omega})$; dotted line, continuation of $S(q, \omega)$, calculated with optimum $G(q, \bar{\omega})$, to frequencies below and above the fitting range; short-dashed line, RPA jellium $S(q, \omega)$; long-dashed line, jellium $S(q, \omega)$, calculated with $G(q, 0)$ of Ref. 7 .

$$
\begin{gathered}
\bar{\omega}=\left[\omega_{<}\left(I_{u}\right)+\omega_{<}\left(I_{l}\right)\right] / 2, \\
\Delta \omega=\left[\omega_{<}\left(I_{u}\right)-\omega_{<}\left(I_{l}\right)\right] / 2,
\end{gathered}
$$

where

$$
\begin{array}{cl}
I_{u}=0.7 I_{\max }, & I_{l}=0.3 I_{\max } \text { for } q \leqslant 2.47 k_{F}, \\
I_{u}=0.55 I_{\max }, & I_{l}=0.2 I_{\max } \quad \text { for } q>2.47 k_{F} .
\end{array}
$$

$\hbar \omega_{<}(I)$ are the energy losses corresponding to $I$ on the low$\omega$ flank of the $S(q, \omega)$ spectrum.

In Figs. 1(a)-1(d), the calculated $S(q, \omega)$ curves, corresponding to the optimum value of $G(q, \bar{\omega})$, are plotted as solid curves within the fitting range $\bar{\omega} \pm \Delta \omega$ together with the experimental spectra. The continuation of the theoretical $S(q, \omega)$ curves, as calculated with the optimum $G(q, \bar{\omega})$, into the frequency range above and below $\bar{\omega} \pm \Delta \omega$, where this optimum LFCF is no longer valid due to its frequency dependence, is plotted as a dotted line in Fig. 2 for two exemplary cases. One can easily verify that these $S(q, \omega)$ curves do not completely fit the high- $\omega$ flank of the experimental $S(\mathbf{q}, \omega)$, in spite of the fact that this flank is influenced neither by the lattice-induced dip nor by the core contributions in the above selected cases. In this way the $\omega$ dependence of $G(q, \omega)$ is demonstrated. (Since only for $1.99 k_{F} \leqslant q$ $\leqslant 2.38 k_{F}$ the high- $\omega$ flank is free both of the lattice-induced dip and of the core contribution, we have renounced fitting at this flank.) One can see from Figs. 1(a)-1(d) that the diplike lattice-induced fine structure is always outside the frequency range for fitting at the low- $\omega$ flank.

\section{RESULTS AND DISCUSSION}

In Fig. 3(a), the values of the real and the imaginary part of the semiempirical frequency-averaged LFCF, $G(q, \bar{\omega})$, are plotted, as obtained by the above described fitting procedure,
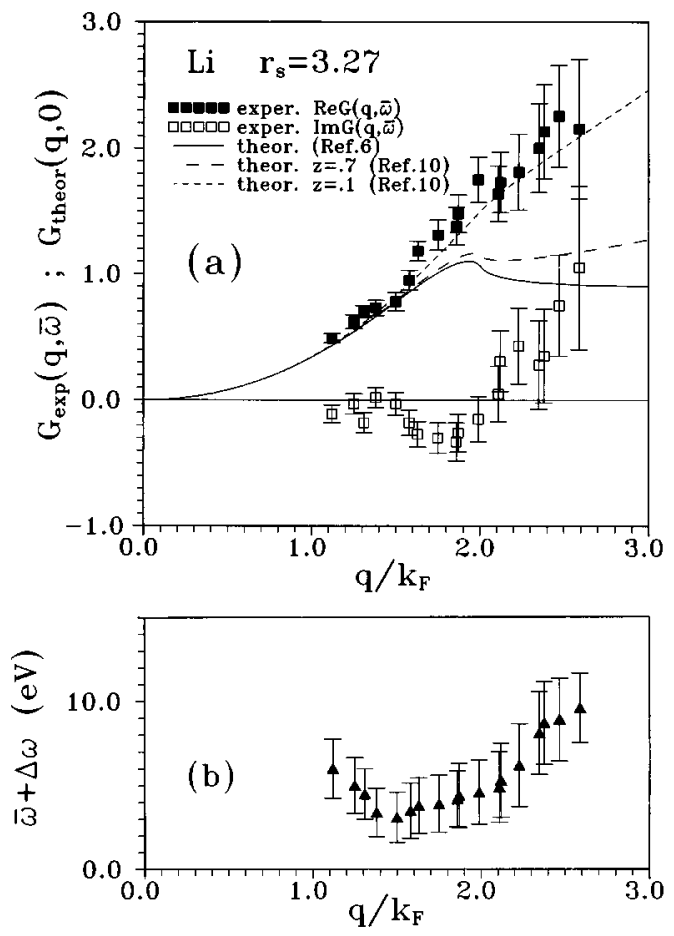

FIG. 3. (a) Data points: semiempirical $G(q, \bar{\omega})$; full squares, $\operatorname{Re} G(q, \bar{\omega})$; open squares, $\operatorname{Im} G(q, \bar{\omega})$; solid line $G(q, 0)$ of Ref. 7; dashed lines, theoretical $G(q, 0)$ according to Ref. 11 using a oneparameter $(z)$ model $-n_{I}(k)$; long dashed line, $z=0.7$; short-dashed line, $z=0.1$. (b) Variation of the fitting frequency range $\bar{\omega} \pm \Delta \omega$ with $q$.

together with several theoretical $G(q, 0)$ curves. In Fig. 3(b) the variation of the fitting range $\bar{\omega} \pm \Delta \omega$ with $q$ is plotted. The error bars on $\operatorname{Re} G(q, \bar{\omega})$ and $\operatorname{Im} G(q, \bar{\omega})$ in Fig. 3(a) are based on an estimated error of $\pm 0.3 \mathrm{eV}$ for the determination of $\omega_{<}(I)$ from the experimental $S(\mathbf{q}, \omega)$ curves. One can easily verify by looking at the error bars of $G(q, \bar{\omega})$ that fitting of experimental $S(\mathbf{q}, \omega)$ for $q>2.5 k_{F}$ will not deliver reasonable results, since the sensitivity of $S(\mathbf{q}, \omega)$ against variations of $G(q, \bar{\omega})$ is rapidly decreasing with increasing $q$. In order to demonstrate the sensitivity of $S(q, \omega)$ against the local-field correction, $S(q, \omega)$ for $G(q, \omega)=0$ are plotted in Figs. 1(a)-1(c) and in Fig. 2 for selected $q$ values (shortdashed curves).

For $q \leqslant 1.6 k_{F}$, the semiempirical $\operatorname{Re} G(q, \bar{\omega})$ agree rather well with the theoretical curve of Utsumi and Ichimaru, ${ }^{6}$ which has been exemplarily plotted in Fig. 3(a). Most of the published theoretical static LFCF's are not very far from the Utsumi-Ichimaru curve in that region of $q$, so that also these calculations find confirmation by the experiment. For $q>1.6 k_{F}$ the semiempirical $\operatorname{Re} G(q, \bar{\omega})$ deviates considerably from all existing calculations. ${ }^{22}$ We do not find either a leveling out for $q \rightarrow \infty$, as predicted by theories, which are using the modified Lindhard dielectric function as a reference for defining of $G(q, \omega)$ (for instance, the UtsumiIchimaru calculations ${ }^{7}$ ) nor a strong peak around $q=2 k_{F}$, as claimed by Brosens et $a l .{ }^{4}$ [In Figs. 1(c) and 1(d) the model calculated $S(q, \omega)$, using the Utsumi-Ichimaru $G(q, 0)$, are plotted as long-dashed curves for selected $q$ 's, in order to demonstrate the sensitivity of the experiment to the proper 
choice of $G(q, \bar{\omega})$.] What we found is a dramatic further increase of $\operatorname{Re} G(q, \bar{\omega})$ after passing $q=1.6 k_{F}$, which is much stronger than the weak quadratic increase, as predicted by theories ${ }^{9,11}$ using the unmodified Lindhard dielectric function as reference for defining of $G(q, \omega)$, a definition, we have employed too. As an example for calculations of this type we have plotted in Fig. 3(a) a $G(q, 0)$ curve (longdashed and short-dashed line, respectively), which we got by using Eqs. (30) and (31) of Ref. 11. The parameter $\delta_{2}$ and $\delta_{4}$ were obtained by employing the following model $n_{I}(k),{ }^{23}$ which contains the renormalization factor $z$ as single parameter, and has the correct limiting behavior for large $k$ :

$n_{I}(k)=\left\{\begin{array}{l}(1-a)-(1 / 2)(1-a-z)\left(k / k_{F}\right)^{8} \text { for } k<k_{F} \\ (1 / 2)(1-a-z)\left(k_{F} / k\right)^{8} \quad \text { for } k>k_{F},\end{array}\right.$

where $a$ is determined by the normalization condition:

$$
4 \pi \int_{0}^{\infty} n_{I}(k) k^{2} d k=(4 \pi / 3) k_{F}^{3}
$$

to $a=(9 / 64)(1-z)$.

Only for the extremely small value $z=0.1$ of the renormalization factor, the calculated $G(q, 0)$ curve fits the semiempirical $\operatorname{Re} G(q, \bar{\omega})$ data. But it must be mentioned that this is just the $z$ value, which fitted best $n(k)$ of $\mathrm{Li}$, as has been reconstructed from directional Compton profile measurements in Ref. 23.

Negative values of $\operatorname{Im} G(q, \bar{\omega})$, as found in our fitting procedure, and a zero passage of $\operatorname{Im} G(q, \bar{\omega})$ at certain $(q, \omega)$ values has already been predicted by Holas et al. ${ }^{5}$ The relative large positive values of $\operatorname{Im} G(q, \bar{\omega})$ for our limiting values of $q$ exhibit such a large error bar that one should not draw hasty conclusions out of this result.

Since for reasons, that have been stated in Sec. III, fitting at the high- $\omega$ flank was not possible for the whole range of $q$, we have not got semiempirical information about $G(q, \omega)$ for large $\omega$. Therefore, we could not utilize the relation between $G(q, \omega \rightarrow \infty)$ and $G(q, 0)$, as pointed out in Ref. 24, in order to get additional internal consistency.

In conclusion, we have found strong deviations of a frequency-averaged semiempirical $\operatorname{LFCF}, G(q, \bar{\omega})$, from all current theories for $q>1.6 k_{F}$, which possibly can be traced back to a corresponding strong deviation of the momentum distribution function $n_{I}(k)$ of interacting electrons from those known so far from theoretical calculations for homogeneous systems. To what extent these deviations are connected with the influence of the periodic lattice needs further investigations. It is our hope that these findings may stimulate calculations both of $G(q, \omega)$ and $n(k)$ for inhomogeneous electrons systems of real metals.

\section{ACKNOWLEDGMENTS}

We thank K.-J. Gabriel for his help with the measurements. This work was funded by the German Federal Ministry of Education and Research under Contract No. 05 650WEA.
1 " " $q$ ", in the argument of $S($ " $q$ ", $\omega)$ and $G($ ' $q$, ,' $\omega)$, respectively, is, in general, written in vector notation. When these functions refer explicitly to a homogeneous electron system, the scalar notation is used for " $q$." "Since the final semiempirical LFCF is obtained by fitting to jellium-calculated dynamical structure factors, it is represented using the scalar notation for " $q$."

${ }^{2}$ J. Hubbard, Proc. R. Soc. London, Ser. A 243, 336 (1958).

${ }^{3}$ D. J. W. Geldart and S. H. Vosko, Can. J. Phys. 44, 2137 (1966).

${ }^{4}$ F. Brosens, J. T. Devreese, and L. F. Lemmens, Phys. Rev. B 21, 1363 (1980).

${ }^{5}$ A. Holas, P. K. Aravind, and K. S. Singwi, Phys. Rev. B 20, 4912 (1979).

${ }^{6}$ D. J. W. Geldart and R. Taylor, Can. J. Phys. 48, 167 (1970).

${ }^{7}$ K. Utsumi and S. Ichimaru, Phys. Rev. B 22, 5203 (1980).

${ }^{8}$ G. Niklasson, Phys. Rev. B 10, 3052 (1974).

${ }^{9}$ A. Holas, in Strongly Coupled Plasma Physics, edited by F. J. Rogers and H. E. Dewitt (Plenum, New York, 1987), pp. 463482.

${ }^{10}$ J. Lindhard, K. Dan. Vidensk. Selsk. Mat. Fys. Medd. 28, 1, 8 (1954).

${ }^{11}$ B. Farid, V. Heine, G. E. Engel, and I. J. Robertson, Phys. Rev. B 48, 11602 (1993).

${ }^{12}$ J. Hong and Y. Shim, J. Phys. Condens. Matter 5, 3431 (1993).
${ }^{13}$ P. M. Platzman and P. A. Wolf, Waves and Interactions in Solid State Plasmas (Academic, New York, 1973).

${ }^{14}$ W. Schülke, in Handbook on Synchrotron Radiation, edited by G. Brown and D. E. Moncton (Elsevier Science, 1991), Vol. 3, pp. 565-637.

${ }^{15}$ H. Ehrenreich and M. H. Cohen, Phys. Rev. 115, 786 (1959).

${ }^{16}$ N. Wiser, Phys. Rev. 129, 62 (1963).

${ }^{17}$ W. Schülke, U. Bonse, H. Nagasawa, A. Kaprolat, and A. Berthold, Phys. Rev. B 38, 2112 (1988).

${ }^{18}$ W. Schülke, H. Nagasawa, S. Mourikis, and P. Lanzki, Phys. Rev. B 33, 6744 (1986).

${ }^{19}$ A. G. Eguiluz, A. Fleszar, and J. A. Gaspar, Nucl. Instrum. Methods Phys. Res. B 96, 550 (1995).

${ }^{20}$ P. K. Aravind, A. Holas, and K. S. Singwi, Phys. Rev. B 25, 561 (1982).

${ }^{21}$ A. Berthold, S. Mourikis, J. R. Schmitz, W. Schülke, and H. Schulte-Schrepping, Nucl. Instrum Methods Phys. Res. A 317, 373 (1992).

${ }^{22}$ Deviations of this kind have already been stated by one of the authors in Ref. 18, but have been speculatively discussed in terms of core polarization effects.

${ }^{23}$ W. Schülke, G. Stutz, F. Wohlert, and A. Kaprolat, Phys. Rev. B (to be published).

${ }^{24}$ A. A. Kugler, J. Stat. Phys. 12, 35 (1975). 